\title{
Research on vehicle soft ground passing ability
}

\author{
Heping Wang ,Hai Xue \\ (Beijing Special Vehicle Research Institute) \\ Email:whpzhjh@sina.com
}

Keywords:vehicle, soft ground, passing ability

Abstract: Soft ground passing ability is an important indicator of vehicle maneuvering performance,in this paper,the vehicle characteristic parameters and soil parameters that affect vehicle passing ability,are analyzed,and the vehicle soft ground passing model is established.Some multi axle vehicle passing ability is test in some desert.

\section{Introduction}

Desert, swamp, snow and other terrain are soft and have a great impact on the passing of vehicles. Therefore, it has important significance to study and evaluate the vehicles' performance of passing the soft ground.

\section{The influence factors on vehicle passing ability}

\section{The influence of the vehicle's parameters on passing ability}

The vehicle parameters which affect passing the soft ground include the maximum unit driving force, the lowest speed, the structure of the wheel and the spacing of the various axle wheel. The maximum unit driving force is larger ,the performance passing soft ground is better.The low speed is smaller,the performance passing soft ground is better. If the vehicle's front wheel tread and the axis track equal and have the same tire width,the front wheel track and each wheel tracking coincides, the rear wheel can travel along the front wheel rut,so the rolling resistance is reduced,the performance f passing soft ground is improved.

The wheel structure has a decisive influence on the passing soft ground. The pattern size, structure parameter and air pressure of the tire all have influence on passing the soft ground.Tire pattern has a great influence on the adhesion coefficient,the wide and depth pattern can increase the soil shear area and ground area to ensure a better adhesion performance. Reducing tire pressure and increasing the width of the tire can increase the contact area between the tire and the ground and reduce the subsidence and rolling resistance, improve the vehicle's passing ability.

\section{The influence of soil parameters on the passing ability}

The main oil parameters affecting the passing ability of the wheeled vehicles include the parameters of soil shear strength, soil moisture, soil density, and so on. The soil moisture content mainly affects the shear strength of soil,The difference of soil moisture content has different influence on the vehicle passing ability.The density of the soil is characterized by the soil's solid degree, permeability, and so on. Different stress and strain characteristics of different density are compared. The shear strength of soil is affect by the density of the arrangement of soil particles.Different density of soil has different effects on the vehicle's passing ability. 


\section{Research on the vehicle's passing soft ground ability.}

\section{Driving force calculation}

According to the relationship between the soil and the vehicle system mechanics, the vehicle is driven by the driving force between the mechanism and the soil The desert and swamp soil can be regarded as a gradual plastic soil, which has a large cohesive and internal friction, the force properties can be expressed by Coulomb formula:

$$
\tau=(c+p \operatorname{tg} \phi)\left(1-e^{-j / K}\right)=(c+p \operatorname{tg} \phi)\left(1-e^{-\delta_{x} / K}\right)
$$

$c$ is soil cohesion, $k P a$;

$\phi$ is internal friction angle of soil, $\left(^{\circ}\right)$;

$\delta$ is slip rate.\%;

$p$ is a unit of area connected pressure, $\mathrm{kPa}$;

$K$ is soil shear modulus of deformation, $\mathrm{cm}$;

$x$ is the distance between the front walking mechanism grounding area and the grounding area element.

The shear stress on the ground area of the whole tire is integrated, and the influence of the height of the tire pattern is considered,so the driving force of the whole vehicle can be calculated ,Due to the vertical load and the ground area are different,the total force is obtained by different wheel force added up.Driving force for different wheel:

$$
\begin{aligned}
& H_{i}=\left\{b l_{i} c\left(1+\frac{2 h}{b}\right)+w \operatorname{tg} \phi\left[1+0.64 \frac{h}{b}\right.\right. \\
& \left.\operatorname{arctg}\left(\frac{h}{b}\right)\right] \times\left[1-\frac{j_{0}}{k_{0}}\left(\left(1-e^{\delta / j_{0}}\right)\right]\right.
\end{aligned}
$$

The total driving force:

$$
H=\sum_{i=1}^{n} H_{i} \quad \sum \lambda_{i}=1
$$

$b$ is the tire width,cm;

$\lambda_{i}$ is the different wheel weight distribution coefficient;

$i$ is the vehicle driving shaft number;

$h$ is the tire tread height,cm;

$l_{i}$ is the different axis of tire ground length, cm;

The formula of the grounding length of different tire is:

$$
l_{i}=\sqrt{2 r_{0}\left(r_{0}-r_{k}\right)-\left(r_{0}-r_{k}\right)^{2}}+\sqrt{2 r_{0}\left(r_{0}-r_{K}+Z_{i}\right)-\left(r_{0}-r_{K}+Z_{i}\right)^{2}}
$$

$r_{0}$ is the tire freedom radius, $\mathrm{cm}$;

$r_{K}$ the tire rolling radius , $\mathrm{cm}$; 
$Z_{i}$ is the subsidence depth of different shaft, $\mathrm{cm}$.

\section{Vehicle driving resistance force}

wheel compaction resistance

When the vehicle is driven on soft ground,the driving resistance is mainly caused by the vertical compaction of the soil.This resistance is a function of the driving force and the slip rate,when the driving force and slip rate increases, the vehicle sinking increase,the resistance increases.

According to rut formation theory, the general Bekker formula can be used to the relationship between the expression of soil per unit area of ground subsidence pressure ${ }^{[3]}$ :

$p=k Z^{n}$

The driving resistance caused $Z=\left(\frac{p_{i}+p_{c}}{k}\right)^{\frac{1}{n}}$ by the wheel rut for each:

$$
P_{f}=\int_{0}^{Z} p s d Z=k b \frac{Z^{n+1}}{n+1}
$$

$n$ is the oil deformation index;

$k$ is the oil deformation modulus, $K N / \mathrm{cm}^{n+2}$;

$Z$ is the rut depth ,cm;

$p_{\text {is }}$ the per unit area of ground pressure, $k P a$;

$p_{i}, p_{c}$ is Tire inflation pressure and tire wall stiffness $(\mathrm{kPa})$,

When calculating the vehicle driving force:

$$
Z=Z_{1}+Z_{2}+\mathrm{K}+Z_{m}
$$

Wheel push sand resistance

The effect of the wheel to push the sand resistance is also considered in the desert;The formula for calculating resistance force :

$$
\begin{aligned}
& P_{R}=\frac{b \sin (a+\phi)}{2 \sin a \cos \phi}\left(s z c k_{c}+r z_{2} k_{r}\right) \\
& +\frac{\pi \gamma\left(90^{\circ}-\phi\right)}{540}+\frac{c \pi l^{2}}{180}+c l^{2} \operatorname{tg}\left(45^{\circ}+\frac{\phi}{2}\right)
\end{aligned}
$$

$k_{c}=\left(N_{c}-\tan \phi\right) \cos ^{2} \phi ;$

$k_{r}=\left(\frac{2 N_{r}}{\tan \phi}+1\right)$

$l=z \operatorname{tg}^{2}\left(45^{\circ}-\frac{\phi}{2}\right)$

$\alpha=\arccos \left(1-\frac{2 z}{D}\right)$ 
$N_{c}, N_{r}$ is sand carrying capacity coefficient;

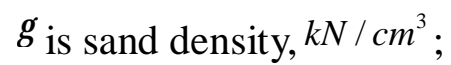

$D$-wheel diameter (cm).

As long as the soil parameters $c$ and $\phi$ are measured at each pass of the wheel,the sand resistance of each wheel can be calculated .By determining the soil parameters and values of the wheel every time, we the resistance force of each wheel can be calculated , and the total resistance force can be calculated.

\section{Vehicle soft ground through performance evaluation}

When the driving force $H$ is greater than the resistance force $\sum P_{f}$, the vehicle can travel. the difference between the two is referred to as the traction force $D P(k N)$, which can be used as the evaluation index of the vehicle passing ability.

$$
D P=H-\sum P_{f}
$$

\section{Test and evaluation of the vehicle passing desert ground ability}

\section{Vehicle operation system characteristics}

The vehicle driving mode can be adjusted, which mainly has $4 \times 4,6 \times 6,8 \times 8$ three driving modes, The protective tire is equipped to the vehicle,the pattern of the tire is non - directional pattern,and central charging and discharging system is also equipped to the vehicle,which can automatically charge and discharge for the tire.The main parameters of the tire see table1.

Table. 1 tire characteristic parameters

\begin{tabular}{|c|c|c|c|}
\hline tire width & pattern height & free radius & tire stiffness \\
\hline $376 \mathrm{~mm}$ & $20 \mathrm{~mm}$ & $653 \mathrm{~mm}$ & $60 \mathrm{ka}$ \\
\hline
\end{tabular}

From the above parameters that the vehicle tire has a deep pattern, the larger the tire diameter and the adjustable tire pressure, these factors determine the ability vehicle has a high ability passing the soft ground.Different axis weight distribution coefficient:

$$
\lambda_{1}=0.25, \lambda_{2}=0.21, \lambda_{3}=0.27, \lambda_{4}=0.27 .
$$

\section{Test schemes}

The soft desert pavement is chosen, its absolute humidity is not more than $4 \%$, the density is $1560 \sim 1670 \mathrm{~kg} / \mathrm{m} 3$, the adhesion coefficient is about 0.65 . Two kinds of schemes are chosen for experiment.

(1)Different tire pressure

For three kinds of pressure test of the vehicle which is equipped with a central adjusting device of the tire pressure, the rated pressure, the minimum allowable pressure and the maximum pressure of the road surface.

(2)Different driving modes

$4 \times 4,6 \times 6,8 \times 8$ three drive modes are respectively driven by means of driving the vehicle's wheel and axle. 


\section{Desert soil parameters test}

Soil pressure characteristic parameters $k$ and $n$ :

Original sand:

$$
k_{0}=1945 \mathrm{kN} / \mathrm{m}^{n+2}, n_{0}=0.97 \text {; }
$$

Compaction two times:

$$
k 2=3856 k N / m^{n+2}, n_{2}=0.78 ;
$$

\section{Vehicle driving force}

If the vehicle is driven in a $8 \times 8$ state, the total driving force is the sum of the tangential traction force of the four axis tires:.

$$
H=H_{1}+H_{2}+H_{3}+H_{4}
$$

When the tire pressure is $0.15 \mathrm{Mpa}$ and $0.3 \mathrm{Mpa}$, the total driving force are:

$$
H_{0.1}=105.1 \times\left[1-\frac{0.49}{71.4 \delta} \times\left(1-\frac{1}{e^{145.4 / \delta}}\right)\right] \quad H_{0.3}=105.1 \times\left[1-\frac{0.49}{85.7 \delta} \times\left(1-\frac{1}{e^{174.8 / \delta}}\right)\right]
$$

The traction force and the slip rate diagram are shown in figure 1 and figure 2:
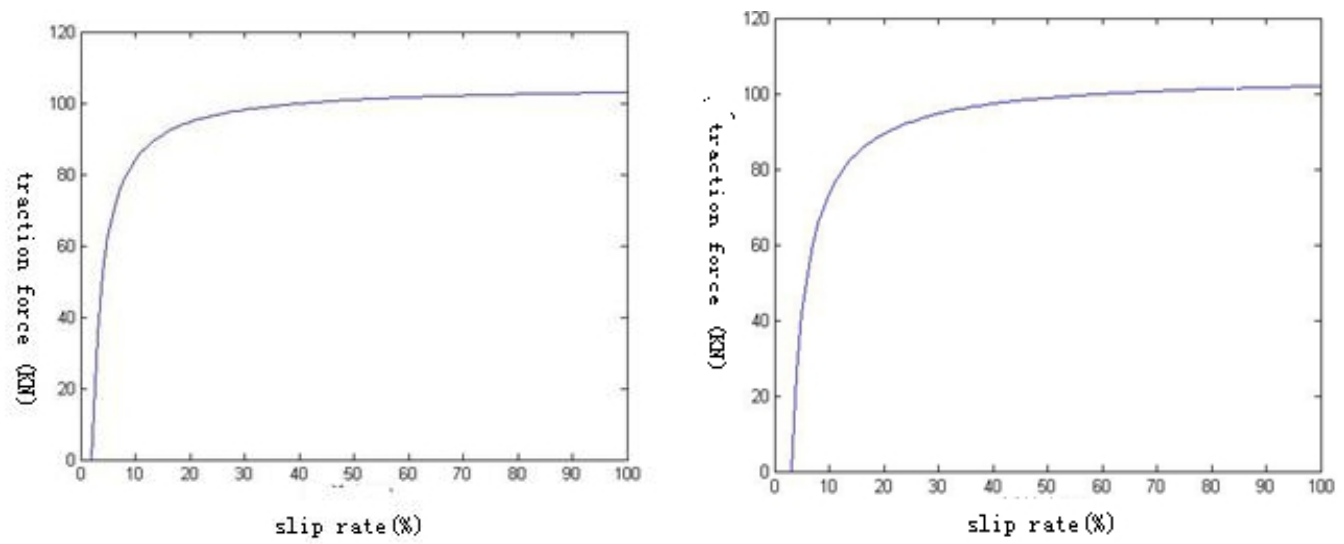

Figure1.relationship between the slip rate and traction force of the tire $0.15 \mathrm{Mpa}$ and $0.3 \mathrm{Mpa}$ pressure Wheel compaction resistance

$$
P_{f}=P_{f 1}+P_{f 2}+P_{f 3}+P_{f 4}=61.6 \mathrm{KN} .
$$

Wheel push resistance

$$
P_{R}=\frac{b \sin (a+\varphi)}{2 \sin a \cos \varphi} y z^{2} k_{r}+\frac{\pi l^{3} \mathrm{Y}\left(90^{\circ}-\varphi\right)}{540}=2.43
$$

Total resistance

$$
P=P_{f}+P_{R}=64.03 k N
$$

\section{Different driving modes of passing ability in the desert}

When the tire pressure is $0.15 \mathrm{MPa}$, the driving mode is $4 \times 4$, the tangential force is:

$$
H=H_{1}+H_{2}=58.4 \times\left(1-\frac{0.49}{71.1 \delta} \times\left(1-\frac{1}{e^{145.4 / \delta}}\right)\right] .
$$

The maximum tangential pulling force is about $54 \mathrm{kN}$, the vehicle resistance is $64.3 \mathrm{kN}$, the vehicle can not pass the desert.

When the tire pressure is $0.15 \mathrm{MPa}$, the driving mode is $6 \times 6$, the tangential force is: 


$$
H=H_{1}+H_{2}+H_{3}=86.5 \times\left(1-\frac{0.49}{71.1 \delta}\left(1-\frac{1}{e^{145.4 \delta}}\right)\right] .
$$

The maximum tangential pulling force is about $54 \mathrm{kN}$, the vehicle resistance is $64.3 \mathrm{kN}$, the vehicle can no pass the desert.,But the slip rate has reached about $26 \%$.

The relationship between the slip rate and the traction force in $4 \times 4$ and $6 \times 6$ model, shown as figure .2 .
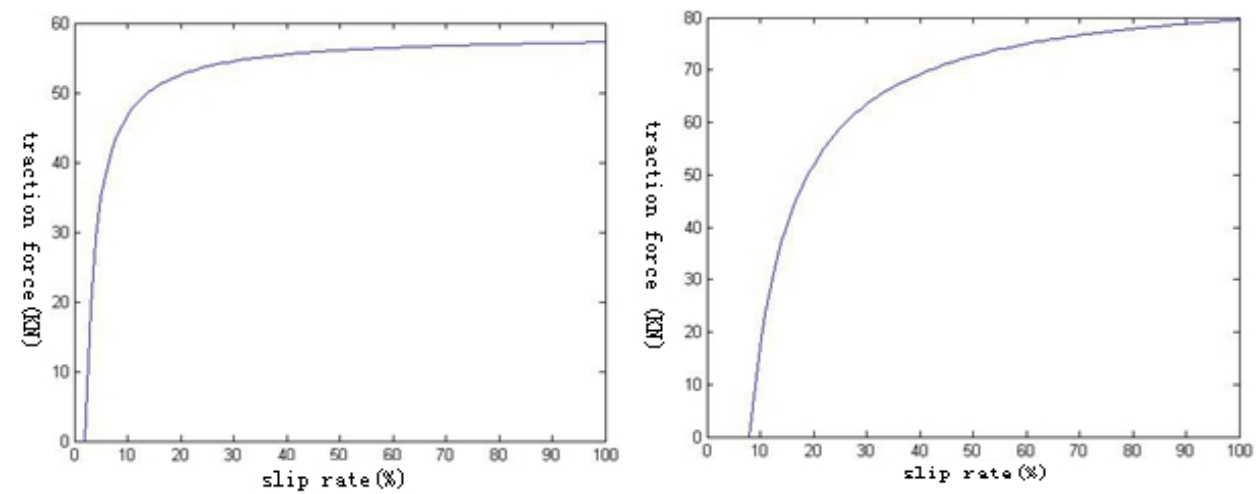

Figure.2. relationship between the slip rate and the traction force in $4 \times 4$ and $6 \times 6$ model

\section{Conclusion}

The tangential traction force of the vehicle increases with the slip rate, and when the slip rate tends to zero, the traction force tends to zero; when the slip rate tends to $100 \%$, the tangential traction force tends to the maximum $105.1 \mathrm{kN}$. With the increase of tire pressure, the vehicle's driving resistance increases, the vehicle slip rate is also increased, the vehicle's desert soft ground by the ability to fall. when the cross country vehicle is driven by a $4 \times 4$ driving way through the sand, the sand resistance is greater than the driving force, which can not pass through the desert region; when the vehicle is passed through the sand by means of $6 \times 6$ driving modes, the driving force is greater than the driving force, Vehicles can pass the desert area.

\section{References}

[1]Zhong Jia Chen; Guo Sheng Yu; small desert shrub harvester through experimental research and effects on sandy land [J]; Anhui Agricultural Sciences; in 2008 03.;

[2] Lu Huaimin, Zhao Zhiguo; experimental study on the capacity of the wetland of engineering vehicles [J]; vehicle and power technology; 2003.03 ;

[3] Yan Xinping Wu Chaozhong, oil monitoring and diagnosis system of information fusion method and model, lubrication and sealing 2001.03; 